\title{
Full polysomnography versus home sleep study: searching for the optimal procedure
}

\author{
H. Teschler*, M. Berthon-Jones**
}

A patient presents to his primary care physician with severe hypersomnia. How do we balance the clinical and economic requirements? One approach is to refer all patients to full polysomnography in the first instance. The waiting list is unacceptable, and the expense is great, but no repeat diagnostic studies are required. At the other extreme, the primary physician sends a patient home with a pulse oximeter. The equipment is cheap and robust, and interpretation is easy, but an unacceptable proportion of sleep disordered breathing, and all other causes of hypersomnia, will be missed [1].

In this issue of the Journal, PARRA et al. [2] compare the cost of limited home respiratory monitoring using the Edentec ${ }^{\mathrm{TM}}$ device, followed by repeated home studies where necessary, with the cost of obligatory full polysomnography. The result is a resounding victory for limited respiratory monitoring: in their sample population this is of the order of three times cheaper, but of comparable accuracy. The authors meticulously address most of the technical limitations of their excellent and timely study, and we need not examine these here. However, there are a number of philosophical limitations to studies of this sort, which are worth some discussion.

The first question concerns the definition of a respiratory event. PARRA et al. [2] have chosen a particularly generous definition for the polysomnographic studies: any discernible reduction in nasal thermistor signal or period of thoraco-abdominal paradoxical motion lasting $\geq 10 \mathrm{~s}$ associated with an arousal or with a cyclic dip in saturation. Consider a patient whose primary cause of hypersomnolence is 30 arousals $\cdot h^{-1}$ due to back pain. All humans have a large increase in airway resistance during sleep, associated with a large increase in respiratory effort, and with a reduction in ventilation $[3,4]$. Therefore, our subject with back pain will have 30 discernible periods of reduction in nasal airflow, associated with 30 events $\cdot \mathrm{h}^{-1}$. Or, to be slightly more provocative, consider a completely normal subject, or a normal subject with a blocked nose [5]. MATHUR and Douglas [6] give the median frequency of electroencephalography (EEG) arousals from nocturnal sleep during first night polysomnography as 21 events $\cdot \mathrm{h}^{-1}$, and 30 events $\cdot h^{-1}$ was a common finding in normals. Our normal subject would therefore have an apnoea/hypopnoea index (AHI) of up to 30 events $\cdot \mathrm{h}^{-1}$. It is essential that normal ranges be established before using superinclusive definitions of hypopnoea. Ultimately, the only way to diagnose extremely mild degrees of obstructive

*Dept of Pneumology, Ruhrlandklinik, Medical Faculty, University of Essen, Germany. **ResMed Ltd, North Ryde, Sydney, Australia. Correspondence: H. Teschler, Ruhrlandklinik, Tüschener Weg 40, D-45239 Essen, Germany sleep apnoea syndrome would be to show an improvement after tracheostomy. We recommend that the increasingly common practice of gradually widening the definition of hypopnea should cease, and instead, symptomatic sleep apnoea syndrome with little to show on polysomnography be assessed by a trial of continuous positive airway pressure (CPAP).

A second philosophical problem is the confounding of the effects of reduced montage and different sensors with the home versus laboratory effect; there is likely to be spuriously lighter sleep and more arousals in the laboratory. Indeed, a likely advantage of home diagnosis is immediately revealed by sleeping for one night in one's own laboratory with all the equipment on: an experience that we strongly recommend to those who have not tried it.

A third problem is revealed by an informative mixing of metaphors. To compare the polysomnography and home results, the authors very reasonably use a Bland and Altman analysis [7]. The metaphor is that the AHI during polysomnography is not the gold standard, but just another fallible estimate of what is happening most nights at home. Douglas et al. [8] have demonstrated that knowing the sleep staging results does not assist the diagnosis if the case-mix has a high probability of simple obstructive sleep apnoea. Conversely, the Barcelona group [2] have, again very commendably, presented receiver operator characteristic curves for sensitivity and specificity for the diagnosis of obstructive sleep apnoea syndrome using a polysomnographic cutoff of 10 events $\cdot h^{-1}$ as the gold standard. It is important to examine which of these metaphors is correct. In our opinion, the reason for using polysomnography is not that it gives a better measurement of the AHI at home; it probably does not [9]. The reason for using polysomnography is that (unlike reduced montage studies, at home or otherwise) it permits the diagnosis of nonrespiratory causes of hypersomnia, and that is the only sense in which it is a gold standard. The real question is not "What is the AHI?" but "Will this patient's quality of life improve with CPAP, oxygen, nasal ventilation, etc.?"; the gold standard is the outcome of the therapy [10].

It is not always possible to reliably differentiate obstructive and central sleep apnoea without using either ribcage plus abdominal movement, or diaphragm electromyograph (EMG), or oesophageal pressure. However, in both cases the subject will go on to CPAP titration, and it will then become apparent whether there are open airway central events, suggesting more ominous aetiology and requiring more complex therapy [11].

A middle pathway misses nobody, but detects most cases of simple obstructive sleep apnoea syndrome quickly. The patient is referred to a specialist out-patient 
clinic, where reasonably detailed unattended home respiratory monitoring is performed, with the object of detecting most cases of sleep disordered breathing [2, 12]. An experienced technician is required to educate the patient and set up the equipment, which is more complex than that for simple oximetry, and a percentage of studies will be lost. A moderately experienced specialist physician is required to interpret the results. The waiting list is moderate, and the cost per test is moderate. If positive, the patient goes on to CPAP titration immediately. One would not contemplate, for example, an irreversible procedure such as uvulopalatopharyngoplasty (UPPP) or surgical mandibular advancement on the strength of this result [13].

At CPAP titration, whether manual [9] or automated $[11,14]$, it will become apparent if the subject has a more complicated disorder of breathing secondary to a stroke, cardiac failure, diseases of the lung and chest wall, or neuromuscular diseases, such as central sleep apnoea, Cheyne-Stokes breathing or rapid eye movement (REM) hypoventilation and hypoxia, which will require oxygen bilevel ventilation, or other highly specialized therapy. If the limited diagnostic procedure is negative, there are many possibilities. Did the patient sleep at all? Did he/she have REM sleep? Did he/she sleep on his back? Was the drug and alcohol level [15], degree of somnolence, and state of the nasal airway typical? Did he/she have bronchospasm? Does the patient have upper airway resistance syndrome with repeated arousals, but not many, desaturations, or hypopnoea [16]? Does the patient have narcolepsy, periodic leg movements (PLMs) [17], or one of the less common parasomnias? The patient then goes on to full polysomnography at a specialist centre.

The split night study, combining diagnosis and initiation of CPAP therapy, has been introduced as an alternative cost containment measure [18]. This has the major disadvantage that it halves the time available for introduction of CPAP, perhaps the most critical moment in management. In addition, the first and second halves of the night are not equivalent [19].

In summary, we provisionally recommend the following management pathway: 1) history and physical examination, with emphasis on the upper airway, and on presence of neurological, cardiac or lung disease, either as the cause of, or secondary to, sleep disordered breathing; 2) if the history and physical examination suggest a high probability of uncomplicated obstructive sleep apnoea, the subject proceeds to unattended home respiratory monitoring, with a minimum of pulse oximetry and semiquantitative nasal airflow; 3) if test results plus history indicate a $90 \%$ probability of simple sleep apnoea, then CPAP is initiated in a semi-attended setting, with the ability to monitor successful normalization of breathing, and the ability to pick up subjects with mouth leak, or residual untreated sleep disordered breathing; 4) if sleep disordered breathing and desaturation is shown to be satisfactory controlled, the subject is sent home on CPAP; and 5) intensive out-patient support during the first 2 weeks with monitoring of symptomatic improvement and compliance.

If the subject fails to meet the clinical criteria at Step 2 , shows complex or equivocal results at Step 3 has continuing sleep disordered breathing or desaturation at Step 4, or has persisting symptoms at Step 5, the subject is likely to have disease processes other than simple obstructive sleep apnoea, and proceeds to polysomnography.
By requiring that symptomatic patients with negative or equivocal studies are always followed up, we miss nobody. By requiring that no irreversible procedure is instituted on the basis of a positive result, we do no harm.

\section{References}

1. Ferber R, Millman R, Coppola M, Fleetham J, Murray $\mathrm{CF}$, Iber $\mathrm{C}$. Portable recording in the assessment of obstructive sleep apnea. ASDA standards of practice. Sleep 1994; 17: 378-392.

2. Parra O, Montserrat JM, Garcia-Esclasans N, et al. Should patients with sleep apnoea hypopnea syndrome be diagnosed and managed on the basis of home sleep studies? Eur Respir J 1997; 10: 1720-1724.

3. Tangel DJ, Mezzanotte WS, White DP. Influence of sleep on tensor palatini EMG and upper airway resistance in normal men. J Appl Physiol 1991; 70: 2574-2581.

4. Dempsey JA, Smith CA, Harms CA, Chow CM, Saupe KW. Sleep-induced breathing instability. Sleep 1996; 19: 236-247.

5. McNicholas WT, Tarlo S, Cole P, et al. Obstructive apneas during sleep in patients with seasonal allergic rhinitis. Am Rev Respir Dis 1982; 126: 625-628.

6. Mathur R, Douglas NJ. Frequency of EEG arousals from nocturnal sleep in normal subjects. Sleep 1995; 18: 330-333.

7. Bland JM, Altman DG. Statistical methods for assessing agreement between two methods of clinical measurement. Lancet 1986; 1: 307-310.

8. Douglas NJ, Thomas S, Jan MA. Clinical value of polysomnography. Lancet 1992; 339: 347-350.

9. Teschler H, Farhat AA, Exner V, Konietzko N, BerthonJones M. AutoSet nasal CPAP titration: constancy of pressure, compliance and effectiveness at 8 months follow-up. Eur Respir J 1997; 10: (in press).

10. American Thoracic Society: Indications and standards for use of nasal continuous positive airway pressure (CPAP) in sleep apnea syndromes. Am J Respir Crit Care Med 1994; 150: 1738-1745.

11. Berthon-Jones M, Lawrence S, Sullivan CE, Grunstein R. Nasal continuous positive airway pressure treatment: current realities and future. Sleep 1996; 19: s131-135.

12. Gugger M. Comparison of ResMed AutoSet (version 3.03) with polysomnography in the diagnosis of the sleep apnoea/hypopnoea syndrome. Eur Respir J 1997; 10: 587-591.

13. Hochbahn W, Brandenburg U, Peter JH. Surgical treatment of obstructive sleep apnea by maxillomandibular advancement. Sleep 1994; 17: 624-629.

14. Teschler H, Berthon-Jones M, Thompson AB, Henkel A, Henry J, Konietzko N. Automated continuous positive airway pressure titration for obstructive sleep apnoea syndrome. Am J Respir Crit Care Med 1996; 154: 734-740.

15. Teschler H, Berthon-Jones M, Wessendorf T, Meyer HJ, Konietzko N. Influence of moderate alcohol consumption on obstructive sleep apnea with and without AutoSet ${ }^{\mathrm{TM}}$ nasal CPAP therapy. Eur Respir J 1996; 9: 2371-2377.

16. Guilleminault C, Stoohs R, Clerk A, Cetel M, Maistros $\mathrm{P}$. A cause of excessive daytime sleepiness. The upper airway resistance syndrome. Chest 1993; 104: 781-787.

17. Yamashiro Y, Kryger MH. Acute effect of nasal CPAP on periodic limb movements associated with breathing disorders during sleep. Sleep 1994; 17: 172-175.

18. Sanders MH, Black J, Costantiono JP, Kern N, Studinicki K, Coates J. Diagnosis of sleep-disordered breathing by half-night polysomnography. Am Rev Respir Dis 1991; 144: 1256-1261.

19. Fanfulla F, Patruno V, Bruschi C, Rampulla C. Obstructive sleep apnoea syndrome: is the "half-night polysomnography" an adequate method for evaluating sleep profile and respiratory events? Eur Respir J 1997; 10: $1725-1729$. 